\title{
Human urinary bladder strip relaxation by the $\beta$-adrenoceptor agonist isoprenaline: methodological considerations and effects of gender and age
}

\author{
Tim Schneider ${ }^{1+}$, Charlotte Fetscher ${ }^{1}$ and Martin C. Michel ${ }^{2 *}$ \\ 1 Departments of Urology and Medicine, University of Duisburg-Essen, Essen, Germany \\ 2 Academic Medical Center, Department of Pharmacology and Pharmacotherapy, University of Amsterdam, Amsterdam, Netherlands
}

Edited by:

Bimal Malhotra, Pfizer, USA

Reviewed by:

Robert James Theobald, A. T. Still

University of Health Sciences-Kirksville

College of Osteopathic Medicine, USA

Margaret A. Vizzard, University of

Vermont College of Medicine, USA

*Correspondence:

Martin C. Michel, Academisch Medisch

Centrum, Afd. Farmacologie en

Farmacotherapie, Meibergdreef 15,

1105 AZ Amsterdam, Netherlands.

e-mail:m.c.michel@amc.nl

${ }^{\dagger}$ Present address:

Praxisklinik Urologie Rhein-Ruhr,

Mülheim, Germany.
The present study was primarily designed to explore various methodological aspects related to organ bath experiments evaluating human detrusor relaxation by the $\beta$-adrenoceptor agonist isoprenaline. Data are based upon a series of 30 consecutive patients, and this cohort was also used to explore possible effects of gender and age. $\mathrm{KCl}$-induced contraction was related to strip length but not weight or cross-sectional area, indicating that the former is most suitable for data normalization. Storage of detrusor strips in cold buffer for up to 2 days did not affect contractile responses to $\mathrm{KCl}$ or efficacy of isoprenaline to cause relaxation but significantly affected the isoprenaline potency. No such alterations were observed with up to 1 day of cold storage. The type ( $\mathrm{KCl}$ vs. passive tension) or strength of contractile stimulus had only minor effects on isoprenaline responses although these differences reached statistical significance in some cases. Similarly, gender and age had only minor if any effects on $\mathrm{KCl}$-induced contraction or isoprenaline-induced relaxation, but the current data are too limited for robust conclusions. In summary we have evaluated experimental conditions for the testing of human detrusor strip contraction and relaxation which should be useful for future larger studies.

Keywords: $\beta_{3}$-adrenoceptor, bladder, human, relaxation, method, age, gender

\section{INTRODUCTION}

Stimulation of $\beta$-adrenoceptors is an important mechanism to increase urinary bladder compliance during the storage phase of the micturition cycle, i.e., the ability to accommodate increasing volumes of urine without major elevation in intravesicular pressure (Andersson and Arner, 2004). $\beta$-Adrenoceptors can potentially exert their compliance-enhancing effects at the level of the urothelium (Masunaga et al., 2010), the afferent nerves (Aizawa et al., 2010), and/or detrusor smooth muscle cells (Michel and Vrydag, 2006). The latter remain the best established contributor to bladder compliance as most functional studies of urinary blad$\operatorname{der} \beta$-adrenoceptors have been based upon relaxation of isolated detrusor strips in an organ bath setting.

Studies with detrusor tissue from animal species have been performed with various types of contractile stimuli, most frequently based on passive tension or contractile responses induced by muscarinic receptor agonists or receptor-independently by $\mathrm{KCl}$. These studies have indicated that the type of contractile stimulus may affect the ability of $\beta$-adrenoceptor agonists to cause relaxation (Longhurst and Levendusky, 1999; Frazier et al., 2005; Michel and Sand, 2009). In contrast, the extent of contractile tone prior to $\beta$-adrenoceptor agonist addition may not importantly affect relaxation responses (Michel and Sand, 2009), but the currently available animal data are too limited for reliable conclusions. Given this uncertainty, it is important to document the strength of contractile stimulus being used for the relaxation experiments. While most investigators agree that such data should somehow be normalized for strip size, they disagree how this should be done. Thus, normalization based upon strip weight (Kories et al., 2003; Monica et al., 2008; Propping et al., 2010), strip length (Schneider et al., 2005a; Frazier et al., 2007), or cross-sectional area (Braverman et al., 2002; Su et al., 2004) have been used, often in the absence of databased justification of the chosen approach. Interestingly, group differences within a study can sometimes be statistically significant when one normalization approach is chosen but not when another is applied (Schneider et al., 2005b).

Studies with detrusor tissue from experimental animals have also indicated that relaxant responses to $\beta$-adrenoceptor agonists may be regulated in several pathophysiological settings; moreover, differences in relaxant responses may also be attributed to physiological differences such as those related to gender or aging, although in most cases such differences were only small (Michel and Barendrecht, 2008).

For all of the above issues, data on isolated human detrusor are much scarcer or even absent. On the other hand, direct extrapolation of the existing larger body of animal data is not necessarily straightforward as relaxation of human detrusor is largely if not exclusively mediated by $\beta_{3}$-adrenoceptors, whereas that of many animal species involves at least partly other subtypes (Michel and Vrydag, 2006). As the three $\beta$-adrenoceptor subtypes differ in their regulation pattern (Curran and Fishman, 1996; Guerrero et al., 1996; Moniotte et al., 2001), specifically studies on physiological or pathophysiological regulation in animals may not necessarily be predictive for the human situation as they may reflect different receptor subtypes. Moreover, many of the functional regulation studies in animals (Michel and Barendrecht, 2008) have been 
performed with agonists which activate all $\beta$-adrenoceptor subtypes, i.e., lack information which specific subtype was regulated functionally. Animal studies with supposedly $\beta_{3}$-selective agonists often are also not conclusive, as many of these compounds lack the expected selectivity for their target receptor (Vrydag and Michel, 2007). Taken together, a relative large body of evidence regarding the function and regulation of rodent bladder $\beta$-adrenoceptors contrasts a much more limited knowledge on the human situation, particularly with regard to regulation under physiological or pathophysiological conditions.

The discrepancy between a desirability of studies with human tissue and its relative lack in the published literature at least partly relates to a limited availability of human tissue to most experimental investigators. The potential ability to use tissue specimen over several days may help to explore more of these questions for human detrusor, as the amount of tissue obtained from a given patient in many cases is not rate limiting. Therefore, the primary aim of the present study was to explore how multi-day storage of human detrusor strips affects contractile responses to $\mathrm{KCl}$ and relaxant responses to the $\beta$-adrenoceptor agonist isoprenaline. As secondary aims we have explored the role of normalization procedures in analysis of human contraction data, the role of contractile stimulus and force for relaxation responses, and finally the roles of gender and age in contraction and relaxation.

\section{MATERIALS AND METHODS PATIENTS}

Human detrusor tissue was from 30 consecutive patients ( 25 males, 5 females; age of $62.7 \pm 1.9$ years, range $31-78$ years) undergoing cystectomy due to bladder cancer from macroscopically tumor-free parts of the bladder after having obtained informed patient consent based upon a protocol approved by the ethical committee of the University of Duisburg-Essen. The specimens were transported to the laboratory within $30 \mathrm{~min}$ after surgical removal and muscle strips were prepared (length $15 \pm 4 \mathrm{~mm}$, weight $21 \pm 1 \mathrm{mg}, n=66$ strips). The cross-sectional area of each strip was calculated based on weight and length assuming a density of $1 \mathrm{mg} / \mathrm{mm}^{3}$ and was $14.8 \pm 0.4 \mathrm{~mm}^{2}$. In some cases, strips were used in the organ bath on the day of surgical removal (day 0 ), whereas in other cases strips were stored in ice-cold buffer for up to 2 days (days 1 and 2).

\section{RELAXATION STUDIES}

The relaxation studies were performed as previously described (Frazier et al., 2011). Briefly, bladder strips were mounted under a resting tension of $10 \mathrm{mN}$ in organ baths containing $10 \mathrm{ml}$ KrebsHenseleit buffer of the following composition (mM): $\mathrm{NaCl} 118.5$, $\mathrm{KCl}$ 4.7, $\mathrm{MgSO}_{4} 1.2, \mathrm{Na}_{4}$ EDTA 0.025, $\mathrm{CaCl}_{2} 2.5, \mathrm{KH}_{2} \mathrm{PO}_{4} 1.2$, $\mathrm{NaHCO}_{3} 25$, Hepes 10 , and glucose 5.5 at $37^{\circ} \mathrm{C}$, yielding a total potassium concentration of $5.9 \mathrm{mM}$. The organ baths were continually gassed with $95 \% \mathrm{O}_{2} / 5 \% \mathrm{CO}_{2}$ to maintain a $\mathrm{pH}$ of 7.4 . The bladder strips were equilibrated for approximately $75 \mathrm{~min}$ during which the buffer solution was refreshed every $15 \mathrm{~min}$. Following the equilibration, the tissues were challenged with $50 \mathrm{mM} \mathrm{KCl}$ for $6 \mathrm{~min}$ and washed again with fresh buffer. They were again equilibrated and readjusted to a baseline tension of $10 \mathrm{mN}$ every 10 min until stabilization had occurred, usually within $45 \mathrm{~min}$. After $10 \mathrm{~min}$ strips were pre-contracted with $50 \mathrm{mM} \mathrm{KCl}$ unless otherwise indicated. After $30 \mathrm{~min}$ (20 min after $\mathrm{KCl}$ ), when tension in $\mathrm{KCl}$-pre-contracted strips had reached a plateau, concentrationresponse curves for isoprenaline $(1 \mathrm{nM}-100 \mu \mathrm{M})$ were generated. To avoid desensitization, only a single relaxation curve was generated in each bladder strip. As our previous data show that the contractile response to $\mathrm{KCl}$ declines by less than $5 \%$ over the time course of a typical relaxation experiment with rat or human detrusor strips (Frazier et al., 2005, 2011), no corrections for spontaneous tension run-down were made.

Force of contraction immediately prior to the addition of the first agonist concentration within a given experiment was defined as $0 \%$ relaxation, and a force of contraction of $0 \mathrm{mN}$ was defined as $100 \%$ relaxation. If more than one bladder strip from a given patient was used in a given experiment, the mean of all strips (up to six) was considered as one experiment unless otherwise stated.

\section{CHEMICALS}

Isoprenaline $\mathrm{HCl}$ and all other chemicals were from Sigma-Aldrich (Munich, Germany).

\section{DATA ANALYSIS}

Data are presented as means \pm SEM of $\mathrm{n}$ experiments. Depending on the type of analysis, an experiment could be an individual detrusor strip or the pooled strips (up to six) of a given patient as indicated. The statistical significance of differences in group means was determined by paired and un-paired (depending on type of analysis) two-tailed $t$-tests. The statistical significance of associations between graded parameters was determined by linear regression analysis. A $p<0.05$ was considered as significant. All curve fitting and statistical analyses were performed with the Prism program version 5.1 (GraphPad Software, San Diego, CA, USA).

\section{RESULTS}

\section{KCI-INDUCED CONTRACTION}

We first analyzed how strip size and storage might affect contractile responses to $\mathrm{KCl}$. Using only the 34 strips from 23 patients which had been tested on the day of surgical removal, we did not detect significant correlations between either strip length, weight or calculated cross-sectional area on the one and the magnitude of the $\mathrm{KCl}$ response on the other hand (data not shown). A similar picture was also obtained among the strips tested on day 2 (21 strips from 17 patients) or 3 (11 strips from 7 patients). However, if data from all 66 strips ( 30 patients) from all 3 days were combined, the $\mathrm{KCl}$ response was weakly but significantly associated with strip length but not weight or cross-sectional area (Figure 1). Therefore, all further analyses of $\mathrm{KCl}$ responses were based on length-adjusted force of contraction ( $\mathrm{mN} / \mathrm{mm})$.

We next explored whether storage for up to 2 days affected the contractile response to $\mathrm{KCl}$. If only the strips from the patients with data on day 0 and 1 were considered ( 16 and 15 strips, respectively, from 11 patients) or only those with data on day 0 and 2 were considered (10 and 10 strips, respectively, from six patients), all groups exhibited very similar contractile responses (Figure 2). Hence, cold storage for up to 2 days did not affect contractile responses to $\mathrm{KCl}$.

While $\mathrm{KCl}$ responses were numerically about a third lower in strips from female as compared to male patients, the difference did not reach statistical significance with the limited number of 

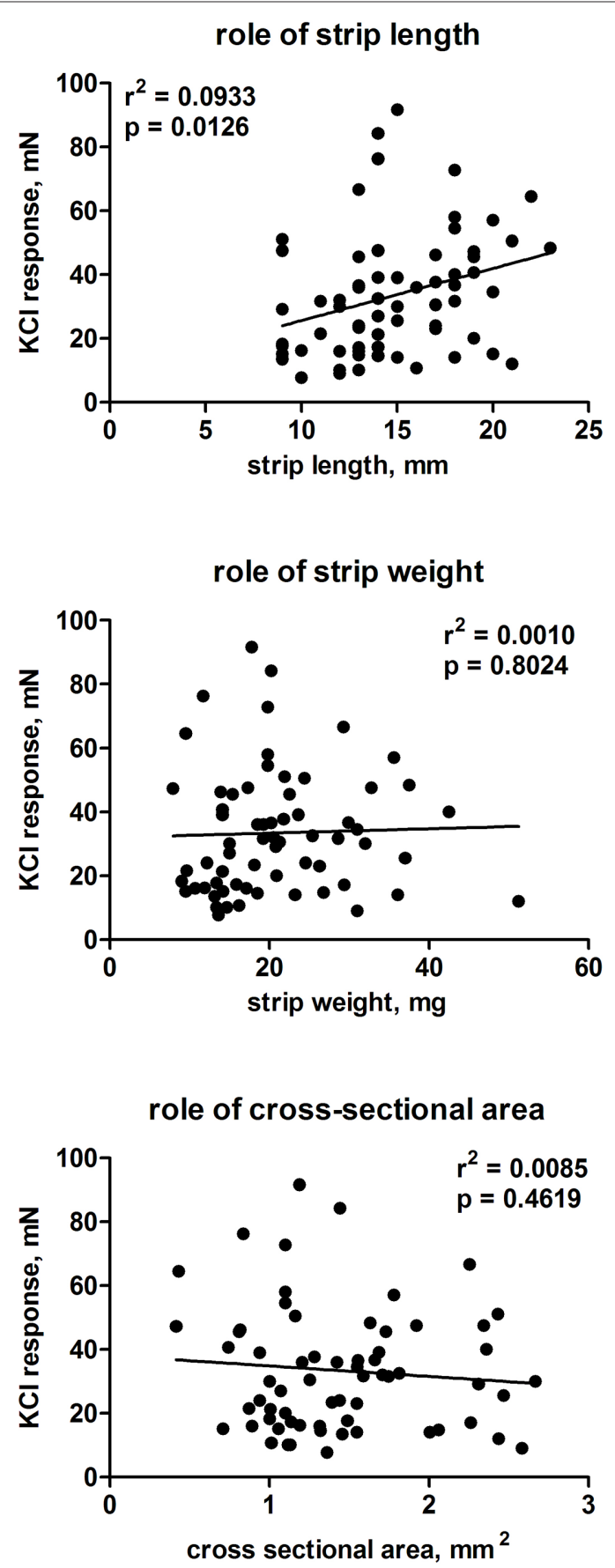

FIGURE 1 | Role of normalization of contractile responses to $50 \mathrm{mM} \mathrm{KCl}$ based upon strip length (upper panel), weight (middle panel), or calculated cross-sectional area (lower panel). Each data represents one strip (in total 66 strips from 30 patients), and data obtained on days 0-2 were included.

patients (Figure 3). Pooling data from both genders, $\mathrm{KCl}$ responses tended to decrease with age but this also failed to reach statistical significance (Figure 3). If only male patients were considered, the association between age and contraction also lacked statistical significance $\left(r^{2}=0.0349 ; p=0.1891\right)$. However, in both cases the day 0 vs. day 1

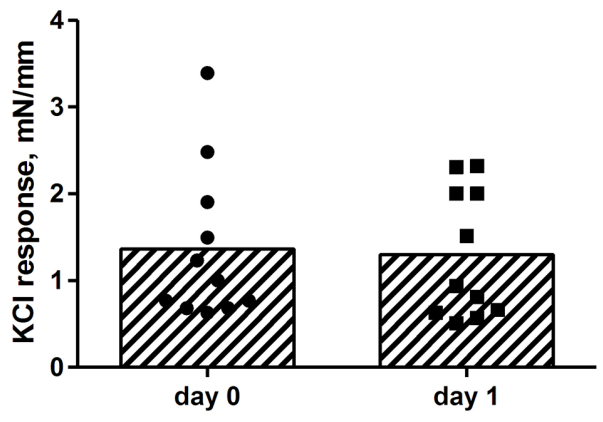

day 0 vs. day 2

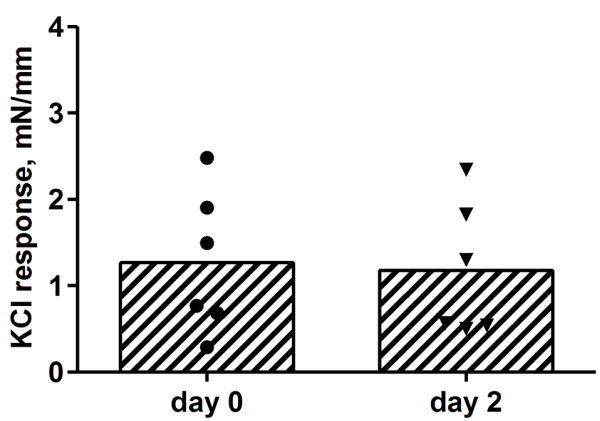

FIGURE 2 | Effect of storage on contractile responses to $50 \mathrm{mM} \mathrm{KCl}$. Strips were tested on the day of surgical removal (day 0 ) or 1 or 2 days later (day 1 and 2). Each data point represents one patient (16 and 15 strips, respectively, from 11 patients for the day 0 vs. 1 comparison; 10 and 10 strips, respectively, from 6 patients for the day 0 vs. 2 comparison), whereas the bars represent the group means. Note that the day 0 patients in the upper panel are not necessarily the same as in the lower panel, as each panel only shows patients for whom data on both days were available.

youngest patient, who had a very small response, appeared to affect the correlation analysis; if he was excluded from the analysis, the inverse association between age and $\mathrm{KCl}$ response remained very weak but became statistically significant $\left(r^{2}=0.0934 ; p=0.0133\right)$ in the pooled group of both genders.

\section{ISOPRENALINE-INDUCED RELAXATION}

Isoprenaline concentration-dependently relaxed $\mathrm{KCl}$-contracted human bladder strips (Figure 4). We first explored whether storage for up to 2 days affected the relaxant response to isoprenaline. If only the strips from the patients with data on day 0 and 1 were considered (16 and 15 strips, respectively, from 11 patients), the potency and efficacy of isoprenaline were very similar and the difference not statistically significant $(p=0.5879$ and 0.2391 , respectively, in a paired $t$-test; Figure 5). A different situation was observed when looking at patients with data on day 0 and 2 (10 and 10 strips, respectively, from six patients). While storage for 2 days did not affect isoprenaline efficacy, it reduced its potency by $0.70 \mathrm{log}$ units ( $p=0.8275$ and 0.0017 , respectively; Figure 5). Hence, storage for up to 1 day did not affect relaxant responses, whereas 2-day storage maintained isoprenaline efficacy but reduced its potency. Accordingly, only strips from days 0 and 1 were included in the subsequent analyses. 


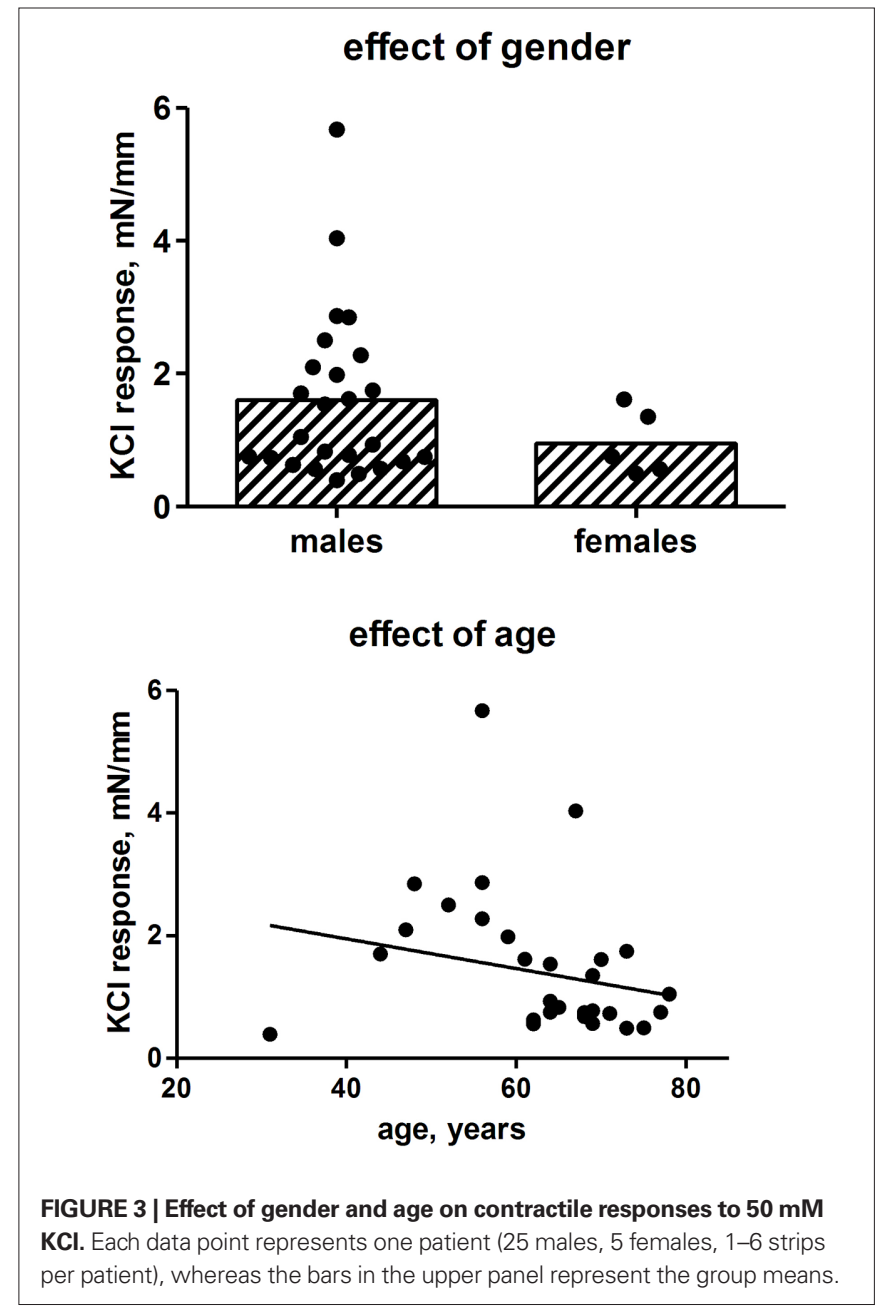

per patient), whereas the bars in the upper panel represent the group means.

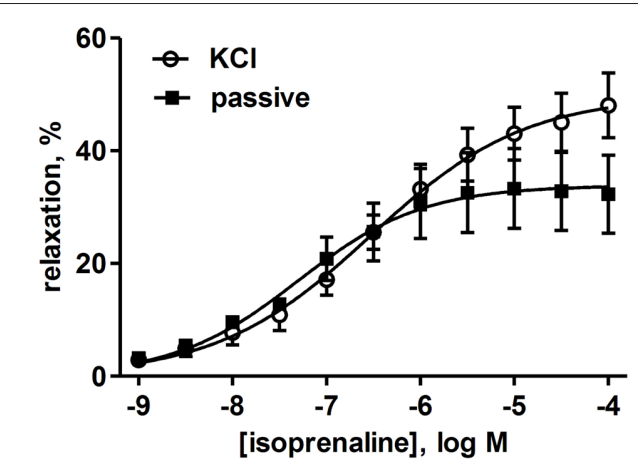

FIGURE 4 | Relaxation of human detrusor strips by isoprenaline under passive tension (filled squares) or with tone induced by $\mathrm{KCl}$ (open circles). Data are means \pm SEM of five patients for which both conditions were studied in parallel on day 0 or 1 with 3-4 strips per patient. For quantitative analysis see main text.

We next explored the role of type and strength of contractile stimulus for the isoprenaline-induced relaxation. Paired relaxation data against passive tension $(10 \mathrm{mN})$ and $\mathrm{KCl}$-induced tone as assessed within one patient were available for 16 pairs of strips from five patients as measured on day 0 and 1 . While the potency and efficacy of isoprenaline was numerically higher against passive tension than against $\mathrm{KCl}$ (Figure 4), this difference did not reach statistical significance $(p=0.0832$ and 0.3713 , respectively; Figure 6). We then used the heterogeneity in $\mathrm{KCl}$-induced tension to explore the role of strength of contractile stimulus. Based upon 54 strips from days 0 and 1, the potency of isoprenaline was not significantly related to strength of contractile stimulus $\left(r^{2}=0.0141\right.$; $p=0.3931)$; in contrast, the efficacy of isoprenaline was weakly but significantly associated with contractile tone, i.e., a greater initial tension was correlated with a greater degree of relaxation $\left(r^{2}=0.1651 ; p=0.0023\right.$; Figure 7$)$.

Finally, we used our patient series to explore possible differences in isoprenaline potency or efficacy related to gender or age. The potency and efficacy of isoprenaline to induce bladder strip relaxation did not differ significantly between genders $(p=0.2573$ and 0.2205 , respectively) although efficacy was numerically greater in males (Figure 8). Similarly, there was no significant association between age on the one and isoprenaline potency or efficacy on the other hand $\left(r^{2}=0.0224\right.$ and $p=0.2757$ and $r^{2}=0.0376$ and $p=0.1561$, respectively; Figure 9).

\section{DISCUSSION}

The present study has primarily been designed for methodological validation of techniques to study human isolated detrusor strip relaxation by the $\beta$-adrenoceptor agonist isoprenaline, a response repeatedly characterized as being predominantly if not exclusively mediated by the $\beta_{3}$-subtype (Michel and Vrydag, 2006; Yamaguchi and Chapple, 2007). Our main interest in this regard was whether storage of muscle strips for up to 2 days was possible without affecting contraction or relaxation responses. This was based upon a series of 30 consecutive patients, many of whom were studied on multiple days following surgical removal of the bladder specimens. Findings from these patients were also used to explore effects of gender and age on such relaxation.

As type and strength of contractile stimulus being used prior to adding the relaxing agonist may affect its potency and/or efficacy (Longhurst and Levendusky, 1999; Frazier et al., 2005; Michel and Sand, 2009), we have initially explored optimal conditions of analyzing $\mathrm{KCl}$-induced contraction. Even when great care is applied during the preparation, detrusor strips exhibit some variance in physical dimensions. As this may affect their ability to contract and relax, it is generally agreed that some type of normalization for strips size is required. However, it remains unclear whether strip weight, length or cross-sectional area is most suitable for such normalization. Our previous work in rats had demonstrated that force of contraction correlated to some extent with strip weight, but the association with strip length was much stronger, indicating that strip length rather than weight should be used for normalization (Schneider et al., 2005a). Our present studies in humans demonstrate that strip length is stronger associated with $\mathrm{KCl}$-induced force of contraction than strip weight or cross-sectional area and hence we have used the former for all subsequent normalization.

The primary question of our study had been whether it is possible to store human detrusor strips in cold buffer without relevant alterations of contraction or relaxation. Our data demonstrate that 
$\mathrm{pEC}_{50}$ day 0 vs 1

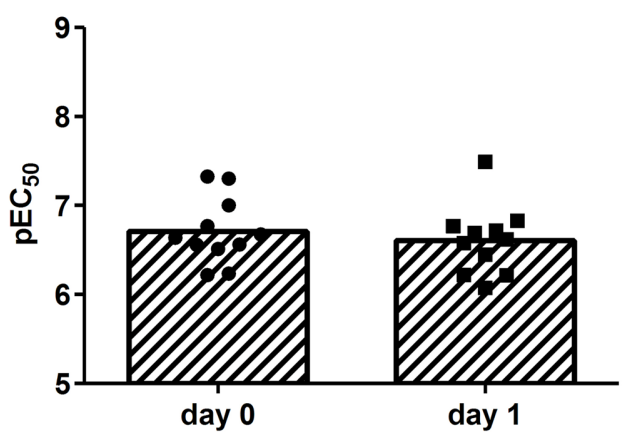

$E_{\max }$ day 0 vs 1
$\mathrm{pEC}_{50}$ day 0 vs 2

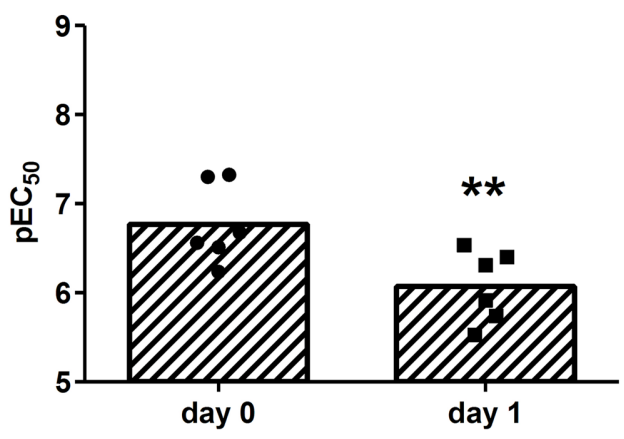

$E_{\max }$ day 0 vs 2

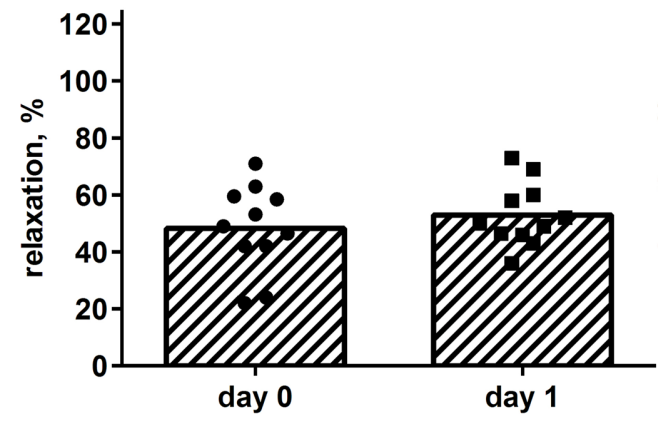

FIGURE 5 | Effect of storage on relaxant responses to isoprenaline, expressed as potency $\left(p \mathrm{EC}_{50}\right)$ and efficacy $\left(E_{\max }\right)$ as calculated per strip. Strips were tested on the day of surgical removal (day 0) or 1 or 2 days later (day 1 and 2). Each data point represents one patient (16 and 15 strips, respectively, from 11 patients for the day 0 vs. 1 comparison; 10 and 10 strips, respectively,

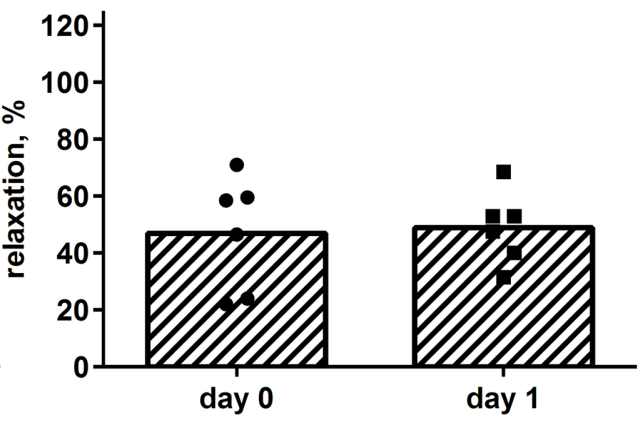

from six patients for the day 0 vs. 2 comparison), whereas the bars represent the group means. Note that the day 0 patients in the upper panel are not necessarily the same as in the lower panel, as each panel only shows patients for whom data on both days were available. ${ }^{*} p<0.05$ in a paired, two-tailed t-test vs. corresponding data from day 0 .
$\mathrm{KCl}$-induced contraction is indeed not affected by storage for up to 2 days. Similarly, cold storage for up to 3 days did not affect contractile responses of isolated arteries to strong stimuli (Kawaguchi et al., 2010). While the potency and efficacy of isoprenaline to cause relaxation also was maintained after cold storage for up to 1 day, storage for 2 days reduced the observed potency of isoprenaline. Hence, for contraction experiments detrusor strip storage for up to 2 days may be appropriate, whereas for relaxation experiments we have limited this to 1 day.

As type or strength of contractile stimulus may affect subsequent relaxation responses in rat detrusor strips (Longhurst and Levendusky, 1999; Frazier et al., 2005; Michel and Sand, 2009), we have explored this for human detrusor. Confirming our previous observations in rat bladder (Frazier et al., 2005; Michel and Sand, 2009), isoprenaline was numerically more potent but less effective as a relaxing stimulus against passive tension as compared to $\mathrm{KCl}$-induced detrusor tone. While these differences did not reach statistical significance in any of these three studies in rats or humans, the consistency of the findings should form a note of caution when comparing findings obtained under the two conditions. In previous studies in rats we had not detected a clear association between strength of contractile stimulus and potency or efficacy of isoprenaline to cause relaxation (Michel and Sand, 2009), but that study was based on group means of five contractile stimuli, i.e., having limited statistical power. In the present study such relationships were explored within a single type of contractile stimulus (50 $\mathrm{mM} \mathrm{KCl}$ ). This did not reveal statistically significant associations with isoprenaline potency, whereas isoprenaline efficacy was slightly but significantly associated with starting tension. These data urge caution when comparing relaxation responses between two groups of patients, e.g., with and without OAB, if the starting tension is not the same in both.

Finally, we have used our patient series to explore possible effects of gender and age on contractile and relaxant responses in human detrusor. Particularly our data with regard to gender should be interpreted with caution in this regard as our patient series contained only five women, and hence was poorly powered to detect gender differences. While isoprenaline efficacy was numerically smaller in female than male samples, this difference did not reach statistical significance. The isoprenaline potency as well as the contractile responses to $\mathrm{KCl}$ also was similar in both genders. Interestingly, the efficacy of isoprenaline had also been numerically smaller in female as compared to male rats in a previous study; while this trend had consistently been observed with four different agonists including isoprenaline, it had reached statistical 


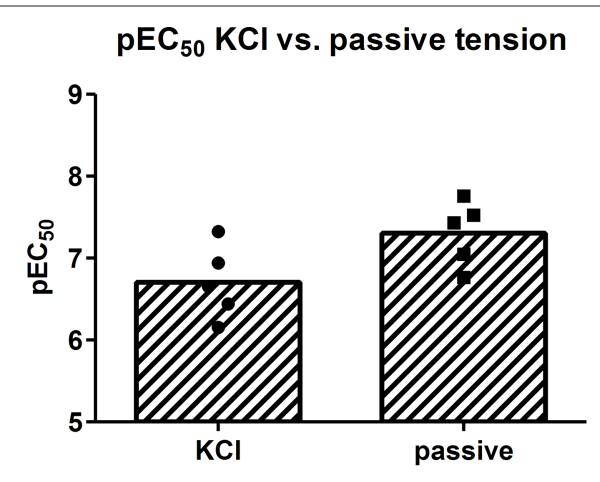

$E_{\max } \mathrm{KCl}$ vs. passive tension

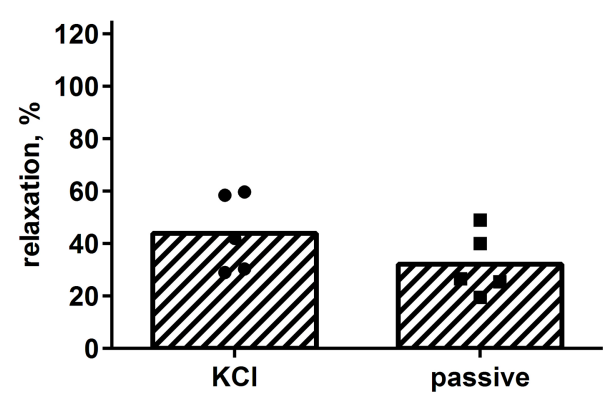

FIGURE 6 | Effect of type of contractile stimulus ( $\mathrm{KCl}$ vs. passive tension) on the potency and efficacy of isoprenaline to relax human detrusor strips. Bars describe group means whereas each data point represents one patient (2-4 strips being tested per patient).
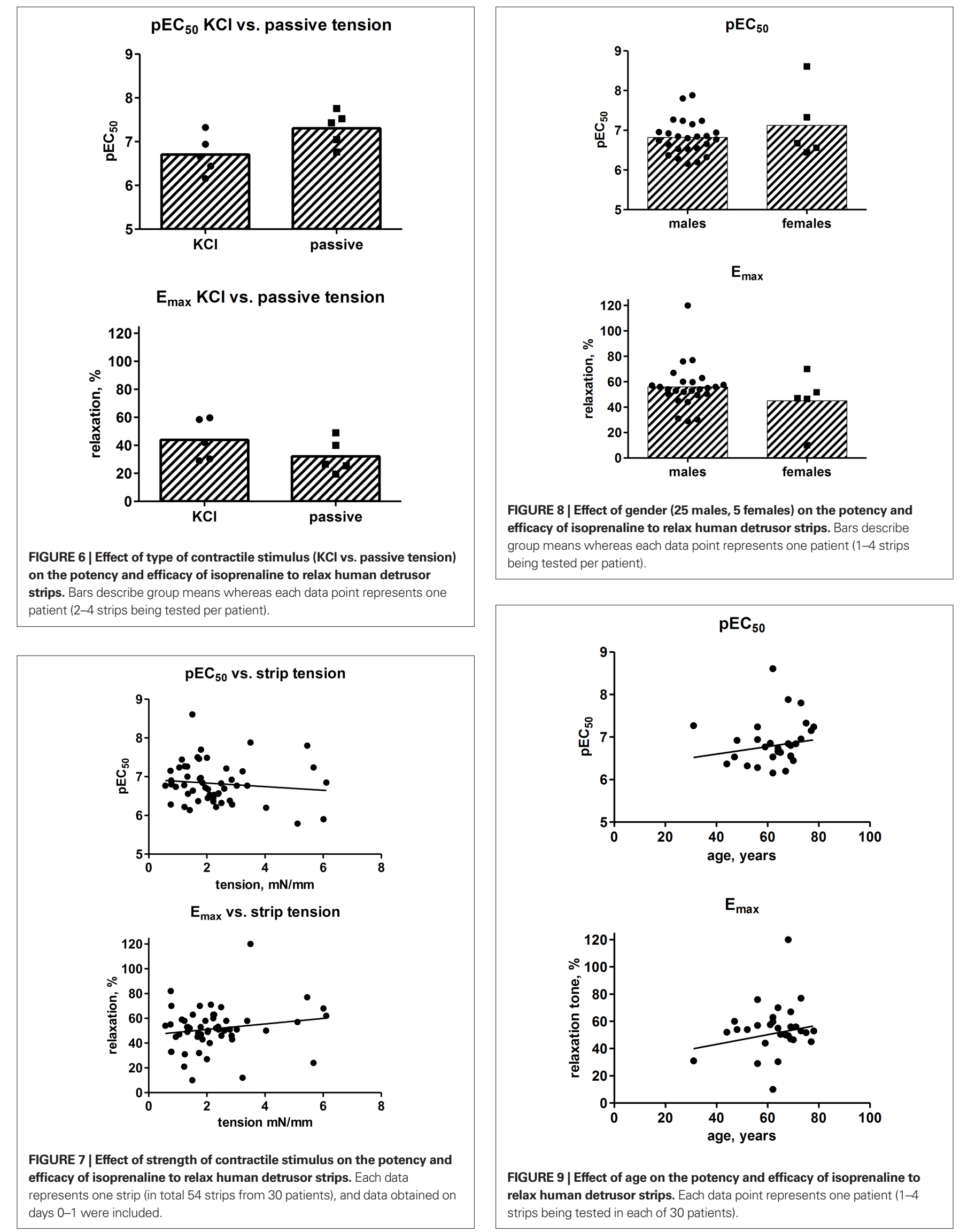

FIGURE 9 | Effect of age on the potency and efficacy of isoprenaline to relax human detrusor strips. Each data point represents one patient (1-4 strips being tested in each of 30 patients). 
significance only for the partial agonists BRL 37,344 and CGP 12,177 (Frazier et al., 2006). On the other hand, a study in rabbits did not detect gender differences in isoprenaline-induced detrusor relaxation, but within that study relaxation responses in the trigone were significantly greater in female than male samples (Morita et al., 1998). Thus, there is insufficient evidence for solid conclusions regarding a possible difference in $\beta$-adrenoceptor-mediated detrusor relaxation.

When exploring possible relationships between age and contraction and relaxation responses in our patient series, we observed that such relationships were weak and on an intentionto-analyze basis not statistically significant. However, the finding that elimination of a single young patient was sufficient to turn this into a "significant" relationship at least for contraction indicates that the present number of samples is insufficient for a robust conclusion. A previous and even smaller study by other investigators had reported a slightly but significantly smaller potency and efficacy to cause human detrusor relaxation for both isoprenaline and BRL 37,344 (Li et al., 2003). In our previous study in rats all four tested agonists were similarly potent in young and old animals; however, their efficacy was consistently smaller in old rats although this did not reach statistical significance for isoprenaline (Frazier et al., 2006). Rat studies by other investigators have also yielded equivocal results (Kolta et al., 1984; Nishimoto et al., 1995; Lluel et al., 2000). Thus, also for the age question the currently available data are insufficient for a reliable conclusion.

In conclusion, our study indicates that human detrusor strips can be stored in cold buffer for at least 1 day without major alterations of isoprenaline potency or efficacy. Our data also emphasize the need for careful consideration of the contractile stimulus being used in relaxation experiments and for detailed reporting of the obtained contraction data. Finally, we conclude that the presently available evidence is too limited to evaluate possible roles of gender or age in the human bladder relaxation by $\beta$-adrenoceptor agonists. Hopefully, the protocols developed here will enable larger studies to answer such questions.

\section{ACKNOWLEDGMENTS}

This work was supported in part through Coordination Theme 1 (Health) of the European Community's FP7, Grant agreement HEALTH-F2-2008-223234. Tim Schneider was a recipient of a training fellowship from the intramural grant programme of the University of Essen Medical School (IFORES).

\section{REFERENCES}

Aizawa, N., Igawa, Y., Nishizawa, O., and Wyndaele, J.-J. (2010). Effects of CL316,243, a $\beta 3$-adrenoceptor agonist, and intravesical prostaglandin E2 on the primary bladder afferent activity of the rat. Neurourol. Urodyn. 29, 771-776.

Andersson, K.-E., and Arner, A. (2004). Urinary bladder contraction and relaxation: physiology and pathophysiology. Physiol. Rev. 84, 935-986.

Braverman, A. S., Tallarida, R. J., and Ruggieri, M. R. Sr. (2002). Interaction between muscarinic receptor subtype signal transduction pathways mediating bladder contraction. Am. J. Physiol. 283, R663-R668.

Curran, P. K., and Fishman, P. H. (1996). Endogenous $\beta 3$-but not $\beta 1$-adrenergic receptors are resistant to agonist-mediated regulation in human SK-N-MC neurotumor cells. Cell Signal. 8, 355-364.

Frazier, E. P., Braverman, A. S., Peters, S. L. M., Michel, M. C., and Ruggieri, M. R. Sr. (2007). Does phospholipase $\mathrm{C}$ mediate muscarinic receptorinduced rat urinary bladder contraction? J. Pharmacol. Exp. Ther. 322, 998-1002.

Frazier, E. P., Mathy, M.-J., Peters, S. L. M., and Michel, M. C. (2005). Does cyclic AMP mediate rat urinary bladder relaxation by isoproterenol? J. Pharmacol. Exp. Ther. 313, 260-267.

Frazier, E. P., Michel-Reher, M. B., van Loenen, P., Sand, C., Schneider, T., Peters, S. L. M., and Michel, M. C.
(2011). Lack of evidence that nebivolol is a $\beta 3$-adrenoceptor agonist. Eur. J. Pharmacol. 654, 86-91.

Frazier, E. P., Schneider, T., and Michel, M. C. (2006). Effects of gender, age and hypertension on $\beta$-adrenergic receptor function in rat urinary bladder. Naunyn-Schmiedebergs Arch. Pharmacol. 373, 300-309.

Guerrero, S. W., Zhong, H., and Minneman, K. P. (1996). Selective desensitization of $\beta 1$ - and $\beta 2$-adrenergic receptors in C6 glioma cells. Receptor 5, 185-195.

Kawaguchi, T., Satoh, K., Kuji, A., and Joh, S. (2010). Features of distinct contractions induced with a high and a low concentration of $\mathrm{KCl}$, noradrenaline, and histamine in swine lingual artery. NaunynSchmiedebergs Arch. Pharmacol. 381, 107-120.

Kolta, M. G., Wallace, L. J., and Gerald, M. C. (1984). Age-related changes in sensitivity of rat urinary bladder to autonomic agents. Mech. Ageing Dev. 27, 183-188.

Kories, C., Czyborra, C., Fetscher, C., Schneider, T., Krege, S., and Michel, M. C. (2003). Gender comparison of muscarinic receptor expression and function in rat and human urinary bladder: differential regulation of M2 and M3? Naunyn-Schmiedebergs Arch. Pharmacol. 367, 524-531.

Li, G., Li, K., Li, Z., and Wang, P. (2003). Age-dependent changes in $\beta$-adrenoceptor function in human detrusor and possible mechanisms. Chin. Med. J. 116, 1511-1514.
Lluel, P., Palea, S., Barras, M. Grandadam, F., Heudes, D. Bruneval, P., Corman, B., and Martin, D. J. (2000). Functional and morphological modifications of the urinary bladder in aging female rats. Am. J. Physiol. 278, R964-R972.

Longhurst, P. A., and Levendusky, M. (1999). Pharmacological characterization of $\beta$-adrenoceptors mediating relaxation of the rat urinary bladder in vitro. Br. J. Pharmacol. 127, 1744-1750.

Masunaga, K., Chapple, C. R., McKay, N. G., Yoshida, M., and Sellers, D. J. (2010). The $\beta 3$-adrenoceptor mediates the inhibitory effects of $\beta$-adrenoceptor agonists via the urothelium in pig bladder dome. Neurourol. Urodyn. 29, 1320-1325.

Michel, M. C., and Barendrecht, M. M. (2008). Physiological and pathological regulation of the autonomic control of urinary bladder contractility. Pharmacol. Ther. 117, 297-312.

Michel, M. C., and Sand, C. (2009). Effect of pre-contraction on $\beta$-adrenoceptor-mediated relaxation of rat urinary bladder. World J. Urol. 27, 711-715.

Michel, M. C., and Vrydag, W. (2006). $\alpha 1$-, $\alpha 2$ - and $\beta$-adrenoceptors in the urinary bladder, urethra and prostate. Br. J. Pharmacol. 147, S88-S119.

Monica, F. Z. T., Bricola, A. A. O., Bau, F. R., Lopes Freitas, L. L., Teixeira, S. A., Muscara, M. N., Abdalla, F. M. F., Porto, C. S., De Nucci, G., Zanesco, A. and Antunes, E. (2008). Long-term nitric oxide deficiency causes mus- carinic supersensitivity and reduces $\beta 3$-adrenoceptor-mediated relaxation, causing rat detrusor overactivity. $\mathrm{Br}$. J. Pharmacol. 153, 1659-1668.

Moniotte, S., Vaerman, J.-L., Kockx, M. M., Larrouy, D., Langin, D., Noirhomme, P., and Balligand, J.-L. (2001). Realtime RT-PCR for the detection of beta-adrenoceptor messenger RNAs in small human endomyocardial biopsies. J. Mol. Cell. Cardiol. 33, 2121-2133.

Morita, T., Masuda, H., Tosaka, A. Ishizaka, K., Tsuhii, T., and Kondo, S. (1998). Sex differences in function and distribution of $\beta$-adrenoceptors in rabbit urinary bladder. J. Urol. 159, 555-558.

Nishimoto, T., Latifpour, J., Wheeler, M. A., Yoshida, M., and Weiss, R. M. (1995). Age-dependent alterations in $\beta$-adrenergic responsiveness of rat detrusor smooth muscle. J. Urol. 153, 1701-1705.

Propping, S., Braeter, M., Grimm, M.-O., Wirth, M. P., Ravens, U., and Wuest, M. (2010). Anticholinergic effects of cis- and trans-isomers of two metabolites of propiverine. NaunynSchmiedebergs Arch. Pharmacol. 381, 329-338.

Schneider, T., Hein, P., Bai, J., and Michel, M. C. (2005a). A role for muscarinic receptors or rho-kinase in hypertension associated rat bladder dysfunction? J. Urol. 173, 2178-2181.

Schneider, T., Hein, P., Michel-Reher, M., and Michel, M. C. (2005b). Effects of ageing on muscarinic receptor subtypes and function in rat urinary 
bladder. Naunyn-Schmiedebergs Arch. Pharmacol. 372, 71-78.

Su, Z., Changolkar, A., Chacko, S., and Moreland, R. S. (2004). Diabetes decreases rabbit bladder smooth muscle contraction while increasing levels of myosin light chain phosphorylation. Am. J. Physiol. 287, F690-F699.

Vrydag, W., and Michel, M. C. (2007). Tools to study $\beta 3$-adrenoceptors.
Naunyn-Schmiedebergs Arch. Pharmacol. 374, 385-398.

Yamaguchi, O., and Chapple, C. R. (2007). $\beta 3$-Adrenoceptor in urinary bladder. Neurourol. Urodyn. 26, 752-756.

Conflict of Interest Statement: The authors declare that the research was conducted in the absence of any commercial or financial relationships that could be construed as a potential conflict of interest.
Received: 06 January 2011; paper pending published: 01 February 2011; accepted: 16 February 2011; published online: 25 February 2011.

Citation: Schneider T, Fetscher $C$ and Michel MC (2011) Human urinary bladder strip relaxation by the $\beta$-adrenoceptor agonist isoprenaline: methodological considerations and effects of gender and age. Front. Pharmacol. 2:11. doi: 10.3389/ fphar.2011.00011
This article was submitted to Frontiers in Cardiovascular and Smooth Muscle Pharmacology, a specialty of Frontiers in Pharmacology.

Copyright $\odot 2011$ Schneider, Fetscher and Michel. This is an open-access article subject to an exclusive license agreement between the authors and Frontiers Media SA, which permits unrestricted use, distribution, and reproduction in any medium, provided the original authors and source are credited. 